\title{
V.
}

\section{Bericht über die Tätigkeit an der Abteilung für Ohren-, Nasen- und Halskranke im k. und k. Garnisonsspitale Nr. 1 in Wien während der Jahre 1900 bis $1902^{1}$ ).}

(Vorstand: Regimentsarzt Privatdozent Dr. Carl Biehl.)

Von

Oberarzt Dr. W. Zemann, Sekundarius der Abteilung.

I. Übersicht der Krankenbewegung in den einzelnen Jahren:

\begin{tabular}{c|c|c|c}
\hline Jahr & Neu zugewachsen & Vom Vorjahr verblieben & Summe \\
\hline \hline 1900 & 326 & 27 & 353 \\
1901 & 325 & 27 & 352 \\
1902 & 375 & 15 & 390
\end{tabular}

Von diesen wurden entlassen:

\begin{tabular}{c|c|c|c|c}
\hline \multirow{2}{*}{ Jahr } & \multicolumn{2}{|c|}{ Dienstfähig } & \multicolumn{2}{|c}{ Dienstunfähig } \\
& Summe & in Prozenten & Summe & in Prozenten \\
\hline 1900 & 208 & 63,80 & 85 & 26,07 \\
1901 & 208 & 64,00 & 100 & 31,07 \\
1902 & 260 & 69,33 & 98 & 26,13
\end{tabular}

Unter den hier als dienstunfähig Bezeichneten sind aber auch jene mit einbezogen, welche nach längerem oder kürzerem Urlaub oder Bädergebrauch wieder als dienstfähig zur Truppe einrückten.

Gestorben sind:

1) Fortsetzung des Berichtes 1898-1899, erschienen in der Monatsschrift für Ohrenheilkunde. 1900. Nr. 2. 


\begin{tabular}{|c|c|c|c|}
\hline Jahr & Summe & In Proz. & Todesursache \\
\hline 1900 & 6 & 1,84 & $\begin{array}{l}1 \text { Fall Miliartuberkulose, } \\
1 \text { Fall Carcinom der Nase, } \\
3 \text { Falle Carcinom des Osophagus, } \\
1 \text { Fall Lues Cerebri. }\end{array}$ \\
\hline 1901 & 2 & 0,62 & $\begin{array}{l}1 \text { Fall Otomeningitis, } \\
1 \text { Fall Durchbruch eines Schläfelappen- } \\
\text { abszesses in die Hirnventrikel. }\end{array}$ \\
\hline 1902 & 4 & 1,06 & $\begin{array}{l}1 \text { Fall Tbe. pulmon. et laryngis, } \\
1 \text { Fall Carcinom. laryngis, } \\
1 \text { Fall Encephalitis, } \\
1 \text { Fall akute Mittelohreiterung, Sinus- } \\
\text { thrombose, Sepsis. }\end{array}$ \\
\hline
\end{tabular}

Die Zahl der Verpflegstage betrug:

$$
\begin{aligned}
& 1900 \text {. . . . . 8464, } \\
& 1901 \text {. . . . . 7002, } \\
& 1902 \text {. . . . } 5956 .
\end{aligned}
$$

Auf einen Kranken entfallen mithin im Durchschnitte im Jahre:

\begin{tabular}{|c|c|c|c|c|c|c|c|c|c|c|c|c|}
\hline Jahr & 苞 & 总 & $\stackrel{\mathbb{3}}{\mathrm{g}}$ & 家 & $\stackrel{\vec{⿷}}{\Sigma}$ & $\stackrel{\vec{E}}{\Xi}$ & 葛 & 獎 & 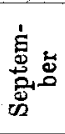 & 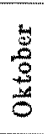 & $\begin{array}{l}\text { Dे } \\
\text { D } \\
0 \\
0\end{array}$ & 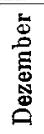 \\
\hline 190 & 3 & 26 & 28 & 31 & 22 & 16 & 10 & 16 & 15 & 73 & 22 & 33 \\
\hline 1901 & $3 t$ & 28 & 24 & 23 & 18 & 16 & 17 & 2 & 16 & 68 & 35 & 22 \\
\hline 1902 & 31 & 24 & 40 & 26 & 25 & 19 & 26 & 20 & 17 & 71 & 36 & 40 \\
\hline
\end{tabular}

$$
\begin{aligned}
& 1900 \text {. . . . 23,97, } \\
& 1901 \text { • • . . 19,89, } \\
& 1902 \text {. . . . 15,27 Verpflegstage. }
\end{aligned}
$$

Der Zugang in den einzeluen Monaten verhielt sich folgendermaaßen:

Wie diese Zahlen, welche mit den an anderen Orten gewonnenen statistischen Beobachtungen nicht tibereinstimmen, zu erklären sind, wurde im ersten Berichte erörtert (Monatssehrift für Ohrenheilkunde. 1900. Nr. 2).

Einige Unregelmäbigkeiten in den Zahlen sind auch da. durch zu erklären, daß die Anzahl jener Kranken immer größer wird, welche von den übrigen Spitälern der Monarchie entweder zur fachärztlichen Behandlung oder in zweifelhaften Fällen zur endgultigen Begutachtung der Abteilung überwiesen werden.

Auber der Behandlung der in Spitalspflege befindlichen Kranken war tiberdies ein Ambulatorium zu versehen, dessen Protokoll im Jahre 
Bericht des k. und k. Garnisonsspitals Nr. 1 in Wien.

$$
\begin{aligned}
& 1900 \text {. . . . 1335, } \\
& 1901 \text {. . . . 1355, } \\
& 1902 \text {. . . } 1751 \text { Besucher aufwies. }
\end{aligned}
$$

Als Ärzte sind tätig auf der Abteilung ein SekundariusOberarzt Dr. Leo Wicherek, dieser wurde anfangs 1902 vom Oberarzt Dr. Z emann abgelöst und ein oder zwei einjährigfreiwillige Mediziner, welche nach Erlangung des Doktorgrades das zweite Halbjahr dienten.

Das Krankenmaterial auch zu Unterrichtszwecken zu verwenden, war reichlich Gelegenheit geboten.

In der im Jahre 1900 neugeschaffenen k. und k. militärärztlichen Applikationsschnle wurde - wie es im ersten Berichte als Wunsch ausgesprochen wurde - erfreulicherweise tatsächlich Ohrenheilkunde als Lehrgegenstand aufgenommen, und mit der Leitung desselben der Chef der Abteilung betraut. Da es nicht möglich ist, die gesamte Schüleranzahl auf einmal zu tubernehmen, so wurde diese in 4 Gruppen gesondert, jede zu höchstens 20 Mann.

Der Unterricht mit einer solchen Gruppe danert 4 Wochen, 2 Stunden täglich.

Über die demselben abgewonnenen Resultate ein Urteil abzugeben, ist einem späteren Berichte vorbehalten.

Ausserdem finden auf der Abteilung noch Universitätskurse 2 pro Semester - statt.

II. Tabellarische Zusammenstellung der Krankheitsformen der in Spitalbehandlung gewesenen Kranken.

a) Ohrenerkrankungen.

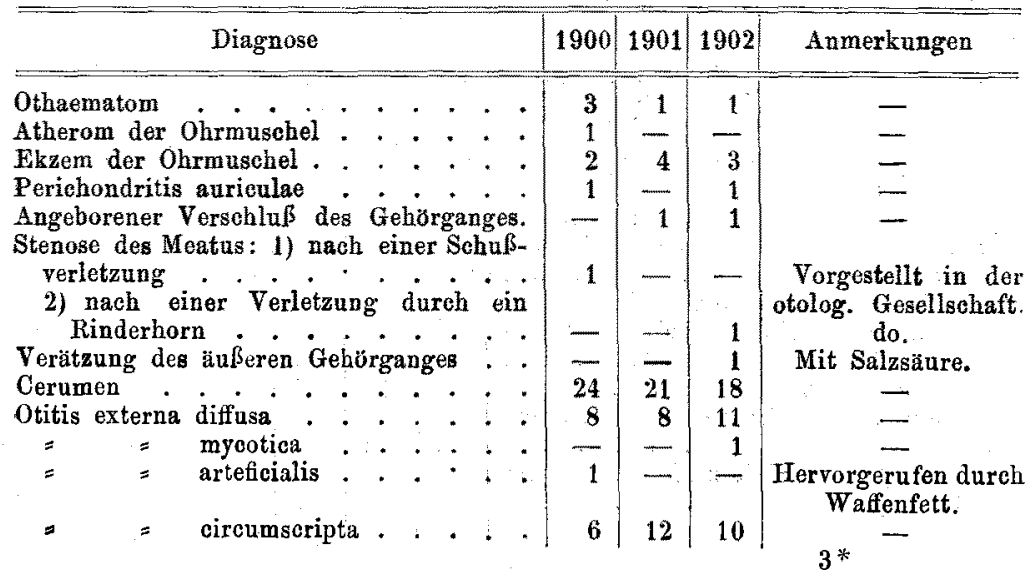




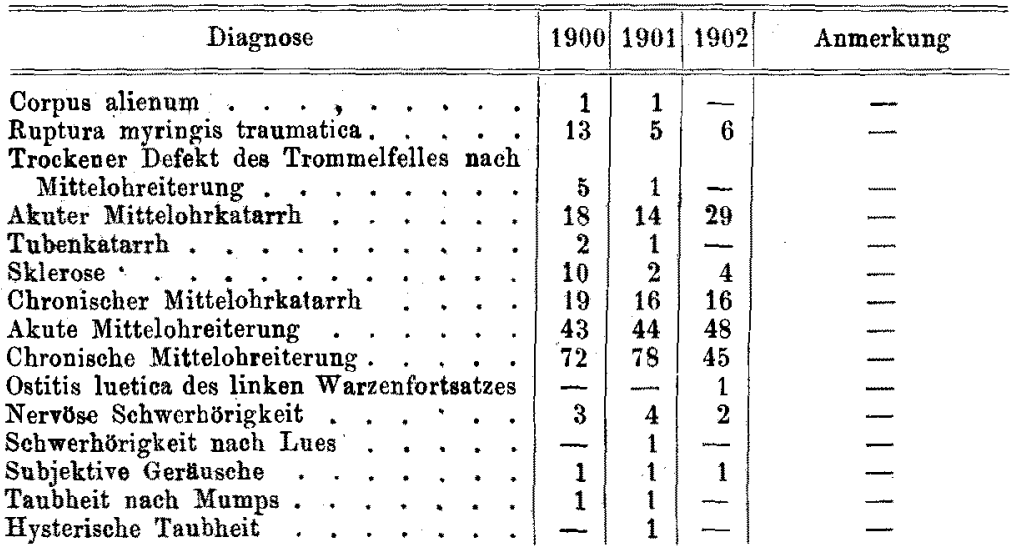

b) Erkrankungen der Nase, des Rachens und des Kehlkopfes.

Ekzem der Nase und des Naseneinganges Carcinom der äusseren Nase. . . . . Tuberkulose der Nase . . . . . . . . Verbiegung der Nasenscheidewand . . . Akuter Nasenkatarrh . . . . . . . . 1 Rhinitis sieca . . . . . . . . . . Atrophischer Nasenkatarrh : . . . . 1 Ozaena . . . . . . . . . . . . Hypertrophischer Nasenkatarrh . . . . 6 Hypertrophischer Katarrh mit polypösen Wucherungen . . . . . . . Lues der Nase . . . . . . . . . . .

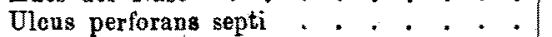
Rhinolith ............. Epistaxis * . * . . . , . . . Empyema sinus frontalis . . . . . . 1 Vordere Kiefercyste . . . . . . . . Entzundung der Oberkieferböhle, akute Entzundung derOberkieferböhle, chronische Polyp der Kieferhöhle - Knöcherne Atresie der rechten Choane
. \begin{tabular}{|c|c|}
1 & - \\
1 & - \\
2 & - \\
1 & $=$ \\
1 & - \\
\hline 1 & 1 \\
\hline 6 & -9 \\
5 & 3 \\
1 & 1 \\
- & - \\
\hline- & - \\
\hline 1 & 4 \\
\hline 1 & 2 \\
- & 2 \\
- & -
\end{tabular}

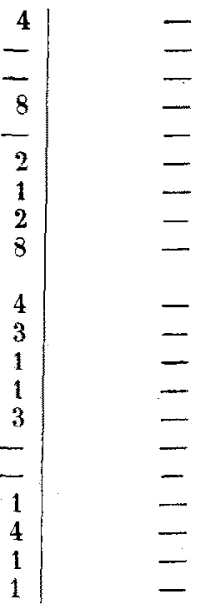
III. Übersicht der Operation.

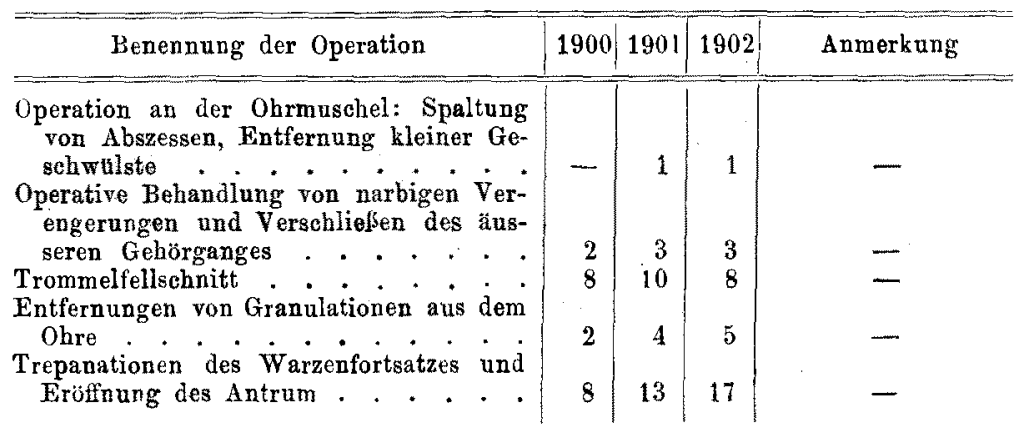




\begin{tabular}{|c|c|c|c|c|}
\hline Benennung der Operation & 1900 & 1901 & 1902 & Anmerkung \\
\hline $\begin{array}{l}\text { Freilegung sämtlicher Mittelobrräume inkl. } \\
\text { der intrakraniellen Komplikationen. (Ex- } \\
\text { traduralabsceß, Sinasthrombose nsw.) }\end{array}$ & 18 & 15 & 11 & - \\
\hline $\begin{array}{l}\text { Plastischer Verschluß einer "retroauricu- } \\
\text { lären" Öffnung }\end{array}$ & 1 & & - & - \\
\hline $\begin{array}{l}\text { Abtragung von hypertrophischer Nasen- } \\
\text { schleimhaut an den Muscheln, sowie } \\
\text { Entfernung der hypertrophischen hinte- } \\
\text { ren Enden }\end{array}$ & 15 & 8 & 16 & - \\
\hline Galvanokaustik der hypertrophischenNasen- & & & & \\
\hline schleimhaut + . . . . . . . & 2 & 8 & 5 & - \\
\hline Entfernung von Nasenpolypen . . . . & 10 & 13 & 11 & - \\
\hline Entfernung yon Choanalpolypen . . & 1 & 1 & 1 & - \\
\hline Abtragung von Spina septi . & 3 & 3 & 4 & - \\
\hline Operationen bei Deviatio septi . . & - & - & 6 & 一 \\
\hline Eutfernung gutartiger Geschwulste der & & & & \\
\hline Nase $\cdot \cdot,+, \cdot, \cdot, \cdot \cdot$ & - & $\mathbf{3}$ & $\mathbf{3}$ & 一 \\
\hline Extraktion von Nasensteinen. $\cdot{ }^{*} \cdot \dot{\text { Pro }}$ & - & - & 1 & - \\
\hline $\begin{array}{l}\text { Eröfnung der Kieferböble durch den Pro- } \\
\text { cessus alveolaris }\end{array}$ & 1 & 2 & 2 & 一 \\
\hline Radicaloperation nach Luc-Caldwell . & - & 1 & 3 & - \\
\hline Operation von Zahncysten. . . . . & 1 & 1 & 1 & 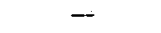 \\
\hline Abtragung von Rachenmandeln. & 37 & 26 & 38 & - \\
\hline Tonsillotomie . . . . . . . . . & 7 & 2 & 11 & - \\
\hline $\begin{array}{l}\text { Spaltung von Tonsillarabszessen . } \\
\text { Operative Entfernung eines Tonsiliar-Fi- }\end{array}$ & $\mathbf{5}$ & 6 & 8 & - \\
\hline bromes . . . . . . & - & - & 1 & - \\
\hline $\begin{array}{l}\text { Kauterisation der Rachenschleimbaut } \\
\text { Abtragung eines Prolapus ventriculi Mor- }\end{array}$ & 1 & 2 & - & 一 \\
\hline 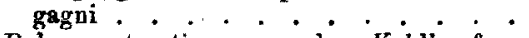 & 1 & - & - & 一 \\
\hline $\begin{array}{l}\text { Polypenextractionen aus dem Kehlkopfe } \\
\text { Laryngofissur. Exeochleation eines Carci- }\end{array}$ & - & 3 & 3 & - \\
\hline noma laryngis + . . . . . . . * & - & - & 1 & - \\
\hline Tracheotomie . . & - & 1 & 1 & - \\
\hline Lumbalpunktionen $\cdot \cdot \cdot \cdot \cdot=$ & - & - & 2 & - \\
\hline
\end{tabular}

Antrumaufmeißelungen im Jahre 1900.

\begin{tabular}{|c|c|c|c|c|c|c|c|}
\hline Nr. & Name & $\begin{array}{c}\text { Auf- } \\
\text { ge- } \\
\text { nom- } \\
\text { men }\end{array}$ & $\begin{array}{l}\text { Ope- } \\
\text { riert }\end{array}$ & $\begin{array}{c}\text { Ent- } \\
\text { lassen }\end{array}$ & 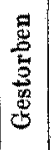 & 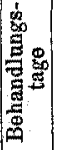 & Bemerkungen \\
\hline 1 & $\begin{array}{l}\text { Anton Uchitil, Unter- } \\
\text { fahrkanonier d. Korps- } \\
\text { Art.-Reg. Nr. 14. }\end{array}$ & $\begin{array}{l}16.1 . \\
1900\end{array}$ & 22. I. & 25. & - & 63 & $\begin{array}{r}\text { Dienstta } \\
n\end{array}$ \\
\hline 2 & $\begin{array}{l}\text { Ilija Krivie, Infant. des } \\
\text { bosn.-herz. Inf.-Reg. } \\
\text { Nr. 1. }\end{array}$ & 12.1II. & 2. $\mathrm{V}$ & 5.VIII. & - & 93 & $\begin{array}{c}\text { Diensttauglich ge- } \\
\text { nesen. }\end{array}$ \\
\hline 3 & $\begin{array}{l}\text { Johann Varga, Infant. } \\
\text { des Inf.-Reg. Nr. } 25 \text {. }\end{array}$ & 15. IV. & 2. V. & $\begin{array}{l}25 . \\
\text { VIII. }\end{array}$ & - & 95 & $\begin{array}{c}\text { Diensttauglich ge- } \\
\text { nesen. }\end{array}$ \\
\hline 4 & $\begin{array}{l}\text { Ferdinand Sersawy, Zögl. } \\
\text { der techn. Mil.-Akad. }\end{array}$ & $\begin{array}{l}11 . \\
\text { VII. }\end{array}$ & $\begin{array}{l}11 . \\
\text { VII. }\end{array}$ & $\begin{array}{l}24 . \text { II. } \\
1901 .\end{array}$ & -1 & 46 & $\begin{array}{l}\text { Diensttauglich ge- } \\
\text { wesen entlassen. }\end{array}$ \\
\hline 5 & $\begin{array}{l}\text { Ignaz Sradi, Husar des } \\
\text { Hus.-Reg. Nr. } 15 .\end{array}$ & $\begin{array}{l}30 . \\
\text { VII. }\end{array}$ & 2.VIII. & 5.VIII. & - & 137 & $\begin{array}{l}\text { Geheilt, aus demHeere } \\
\text { invalid entlassen. }\end{array}$ \\
\hline
\end{tabular}




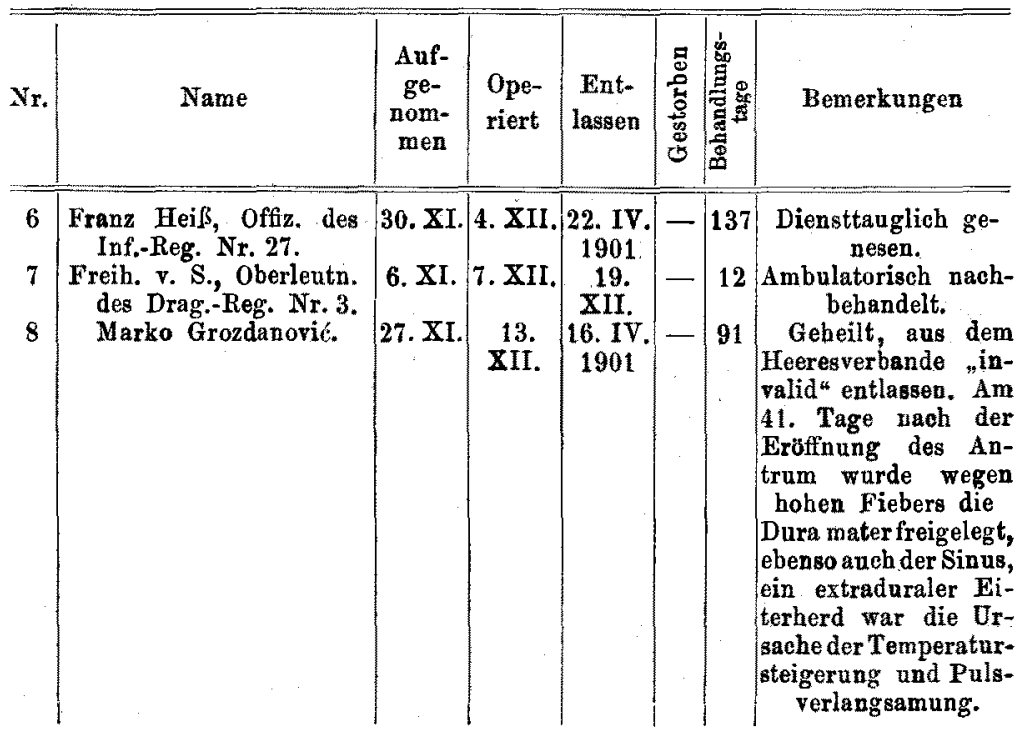

AntrumaufmeiBelungen im Jahre 1901.

\begin{tabular}{|c|c|c|c|c|c|c|}
\hline Frau A. Z. & 15. I. & I. & 23. I. & - & & ah. \\
\hline $\begin{array}{l}\text { Anton Kofol, Infant. d. } \\
\text { Inf,-Reg. Nr. } 8 .\end{array}$ & 19. I. & 22. I. & $31 . \nabla$. & - & 132 & Geheilt, \\
\hline $\begin{array}{l}\text { Nicolaus Morarin, Corpo- } \\
\text { ral d. Inf.-Reg. Nr. } 43 .\end{array}$ & 22. II. & 28. II. & 31. V. & - & 98 & $\begin{array}{l}\text { Erisipelas. } \\
\text { natl. Urlaubs- } \\
\text { entlassen. }\end{array}$ \\
\hline $\begin{array}{l}\text { Max.Hubalek, Zoogl. der } \\
\text { Pion.-Kad.-Schule. }\end{array}$ & 8. III. & $11 . \mathrm{III}$ & $20 . \mathrm{IV}$. & - & 71 & $\begin{array}{c}\text { Diensttauglich ge- } \\
\text { nesen. }\end{array}$ \\
\hline $\begin{array}{l}\text { Johann Stanzig, Zögl. } \\
\text { der Pion.-Kad.-Schule. }\end{array}$ & 26. II. & 24.111 & 20. IV. & - & 53 & $\begin{array}{c}\text { Diensttauglich ge- } \\
\text { nesen. }\end{array}$ \\
\hline $\begin{array}{l}\text { Ibor A bas, Infant, des } \\
\text { bosn.-herz. Inf.-Reg. } \\
\text { Nr. } 1 \text {. }\end{array}$ & 28.111. & 23. IV & 15. V. & - & 79 & $\begin{array}{c}\text { Diensttauglich ge- } \\
\text { nesen. }\end{array}$ \\
\hline $\begin{array}{l}\text { Marko Bebic, Infant. d. } \\
\text { bosn.-herz. Inf.-Reg. } \\
\text { Nr. } 4 .\end{array}$ & 22. IV. & 27. IV & 31. V. & - & 39 & $\begin{array}{r}\text { Diensttan } \\
\text { nes }\end{array}$ \\
\hline $\begin{array}{l}\text { Franz Micbaly, Infant. } \\
\text { des Inf.-Reg. Nr. } 84 .\end{array}$ & $\begin{array}{l}30 . \\
\text { VIII. }\end{array}$ & 9. IX. & $20 . \mathrm{XI}$. & - & 82 & $\begin{array}{c}\text { Geheilt, } m \\
\text { Urlaubsa } \\
\text { las }\end{array}$ \\
\hline Hauptm. Emil J & $13 . I X$ & $\begin{array}{l}\text { 14. u. } \\
\text { 18. IX }\end{array}$ & 3. & - & 20 & $\begin{array}{l}\text { Operat beiderseitig. } \\
\text { Ambulatorisch nach- } \\
\text { behandelt. }\end{array}$ \\
\hline$F_{x}$ & 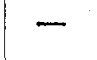 & 18 & $\ldots$ & - & -1 & Ambulatorisch nach- \\
\hline $\begin{array}{l}\text { Unterpionier } \\
\text { Karnel }\end{array}$ & 22. X. & 23.X. & $\begin{array}{l}22 . \\
\text { XII. }\end{array}$ & - & 61 & $\begin{array}{l}\text { Freilegung des Sinus. } \\
\text { Sinusblutang. Mit } \\
4 \text { wöhentl. Urlaubs- } \\
\text { antrag entlassen. }\end{array}$ \\
\hline lleutnant & 23.X. & $23, \mathrm{X}$ & 9. XI. & - & 17. & Erö \\
\hline
\end{tabular}




\begin{tabular}{|c|c|c|c|c|c|c|c|}
\hline $\mathrm{Nr}$. & Name & $\begin{array}{c}\text { Auf- } \\
\text { ge- } \\
\text { nom- } \\
\text { men }\end{array}$ & $\begin{array}{l}\text { Ope- } \\
\text { riert }\end{array}$ & $\begin{array}{c}\text { Ent- } \\
\text { lassen }\end{array}$ & $\mid \begin{array}{l}: \\
8 \\
0 \\
0 \\
0 \\
0 \\
0 \\
0\end{array}$ & 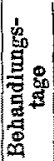 & Bemerkungen \\
\hline 13 & $\begin{array}{l}\text { Oberstleutnant J. Sch. } \\
\text { F. v. B. }\end{array}$ & 2. XI. & 2. XI. & 4. & XI. & 4 & $\begin{array}{l}\text { Operation in ultimis } \\
\text { ohne Narkose im be- } \\
\text { wustlosen Zustande. } \\
\text { Der Sinus wurde frei- } \\
\text { gelegtund eröfnet. - } \\
\text { Sektionsbefund: Ab- } \\
\text { scess im 1. Schläfen- } \\
\text { lappen. Dureh- } \\
\text { brueh desselben in die } \\
\text { Seitenventrikeln. }\end{array}$ \\
\hline
\end{tabular}

Antrumaufmeißelungen im Jahre 1902.

1 Philipp Wittmann, Zögl. $\mid$ 25. I. $\mid$ 27. I. $\mid$ 29. V. $\mid$ - 124|Pat. erkrankte kurz der Inf.-Kad. Schule.

Oberleutn. E. F.

Jaroslar Buchta, Zögl. der Inf.-Kad.-Schule.

4 Regimentsarzt Dr. F. A.

5 Ulan Franz Palywoda.

6 Privater E. F.

7 Jokann Turk, ErsatzReserv. des Inf.-Reg, Nr. 4.

8 Private E. Z.

9 Private T. Z.

10 Infant. Vule Boljanović.

11 Privater F. R.

12 Infant. Johann Bartusek.

3 Oberstenssohn A. B.

14 Privater C. S.

15 Baronesse M, K.

16 Husar Istran Fejes danten M. S.

ror Vernarbung der Operationswunde an Rheumatismus articularum m. Endocarditis. Superarbitriert entlassen.

14. I. 12. II. 22.VII. -

169 Geheilt entlassen, nachdem am 4. März d. Ausräumung sämtlich. Mittelohrräume ausgefuhrt wurde.

56 Geheilt entlassen.

27. II. 4.III. 18.III. -19 Wurde mit Urlaubsantrag entlassen.

25. II. 22. III - 5. - Gestorben an Sepsis, ausgehend von Sinus sigmoideus.

Ambulatoriseh. Geheilt.

Ambulatorisch. Ambulatorisch. Geheilt.

Ambulatorisch. Gebeilt.

Ambulatorisch nach= behandelt. Ambulatorisch.

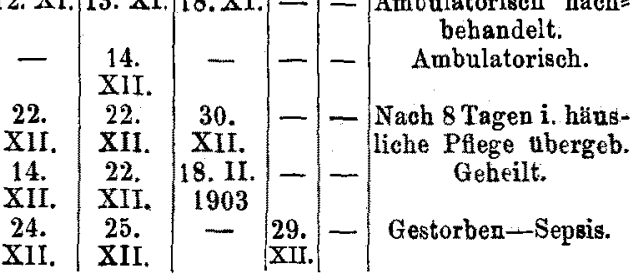


VI. Ausräumung sämtlieher Mittelohrräume im Jahre 1900.

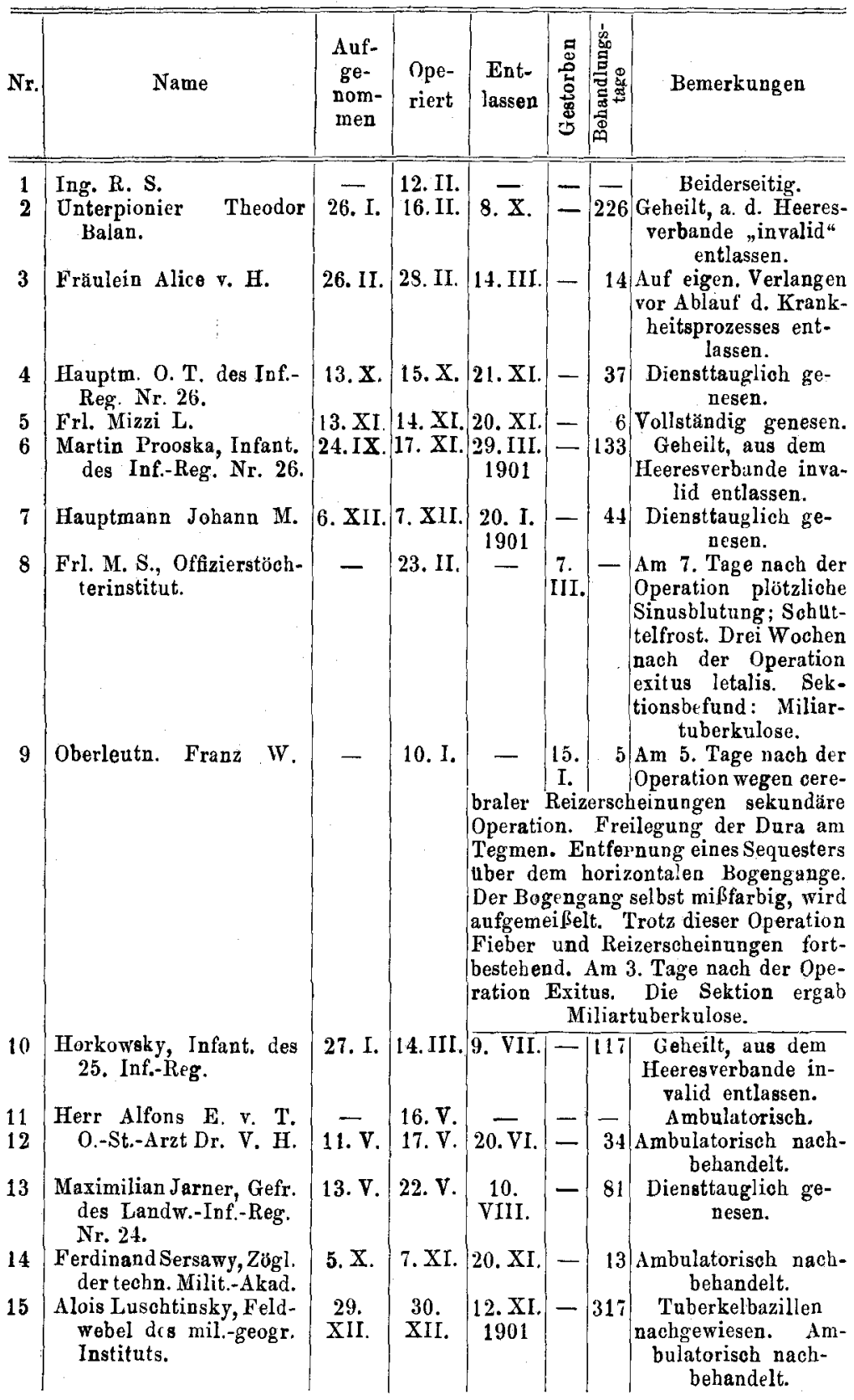


VII. Ausräumung sämtlicher Mittelohrräume im Jahre 1901.

\begin{tabular}{|c|c|c|c|c|c|c|c|}
\hline Nr. & Name & $\begin{array}{l}\text { Auf- } \\
\text { ge- } \\
\text { nom- } \\
\text { men }\end{array}$ & $\begin{array}{l}\text { Ope- } \\
\text { riert }\end{array}$ & $\begin{array}{l}\text { Ent- } \\
\text { lassen }\end{array}$ & 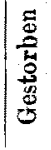 & 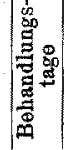 & Bemerkungen \\
\hline 1 & Unterpion. Franz Hutter. & 1. XII. & $\begin{array}{l}9 . \mathrm{I} . \\
1901\end{array}$ & 21. III. & - & 110 & $\begin{array}{c}\text { Superarbitriert ent- } \\
\text { lassen. }\end{array}$ \\
\hline 2 & Frl. L. M. V. B. & 13. I. & 13. I. & 15. II. & - & 33 & $\begin{array}{c}\text { Ambulatorisch nach- } \\
\text { behandelt. }\end{array}$ \\
\hline 3 & $\begin{array}{l}\text { A uton Rattin, Jäger des } \\
\text { 3. 'S.-K.-J.-R. }\end{array}$ & 27. III. & 27. IV. & $\begin{array}{l}20 . \\
\text { VII. }\end{array}$ & - & 115 & $\begin{array}{l}\text { Mit } 8 \text { wöchentlichem } \\
\text { Urlaubsantrag ent- } \\
\text { lassen. }\end{array}$ \\
\hline 4 & $\begin{array}{l}\text { Johann Csupik, Husar } \\
\text { des } 15 . \text { Husaren-Reg. }\end{array}$ & 15. IV. & $12 . \mathrm{V}$. & 5. VI. & - & 51 & $\begin{array}{l}\text { Diensttauglich ge- } \\
\text { nesen entlassen. }\end{array}$ \\
\hline 5 & $\begin{array}{c}\text { Oberleutnant Max H., } \\
\text { Train-Reg. Nr. 1. }\end{array}$ & 31. V. & 1. VI. & 10. VI. & - & 10 & $\begin{array}{l}\text { Cholesteatom. Am- } \\
\text { bulatorisch nach- } \\
\text { behandelt. }\end{array}$ \\
\hline 6 & $\begin{array}{l}\text { Josef Meihers, Husar des } \\
\text { 15. Husaren-Reg. }\end{array}$ & $\begin{array}{l}27 . \\
\text { vii. }\end{array}$ & 14. IX. & XII. & 一 & 149 & $\begin{array}{c}\text { Superarbitriert ent- } \\
\text { lassen. }\end{array}$ \\
\hline 7 & $\begin{array}{l}\text { Anton Nowak, Armee- } \\
\text { dieners-Sohn. }\end{array}$ & 14. IX. & 14. IX. & 25. IX. & - & 11 & 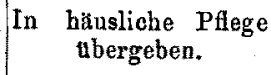 \\
\hline 8 & $\begin{array}{l}\text { Stefan Molnar, Hus. d. } \\
\text { 4. Husarea-Reg. }\end{array}$ & 13.X. & $19 . \mathrm{x}$ & XI. & - & 69 & Zeitlich beurlaubt. \\
\hline 9 & Dr. jur. P. & - & $22 . \mathrm{X}$ & - & - & - & $\begin{array}{c}\text { In häusliche Pflege } \\
\text { ubergeben. }\end{array}$ \\
\hline 10 & $\begin{array}{l}\text { Franz Rabe, Zugsf. des } \\
\text { 3. Inf.-Reg. }\end{array}$ & 7. $\mathrm{x}$. & 26. XI. & $\begin{array}{l}20.1 \mathrm{~V} . \\
1902\end{array}$ & - & 195 & Zeitlich beurlaubt. \\
\hline 11 & $\begin{array}{c}\text { Matthias Glas, Dragon, } \\
\text { des Drag.-Reg. Nr. 4. }\end{array}$ & 10. $X$ & 26. XI. & $\begin{array}{l}10 . \mathrm{V} . \\
1902\end{array}$ & - & 212 & $\begin{array}{c}\text { Superarbitriert ent- } \\
\text { lassen. }\end{array}$ \\
\hline 12 & $\begin{array}{c}\text { Johann Urban, Husar } \\
\text { des 4. Hus.-Reg. }\end{array}$ & 26. XI & $\begin{array}{l}16 . \\
\text { XII. }\end{array}$ & $\begin{array}{l}10 . \text { III. } \\
1902\end{array}$ & - & 103 & $\begin{array}{c}\text { Diensttauglich ge- } \\
\text { nesen. }\end{array}$ \\
\hline 13 & $\begin{array}{c}\text { Franz Stich, Juger des } \\
\text { 21. Feld-Jager-Bat. }\end{array}$ & - & 16. III. & - & - & - & $\begin{array}{l}\text { Naeh } 4 \text { Woohen } \\
\text { wegen bereits fruber } \\
\text { bestehender Epilepsie } \\
\text { auf eine andere Ab- } \\
\text { teilung transferiert. }\end{array}$ \\
\hline 14 & $\begin{array}{l}\text { Ferdinand Sersawy,Zögl. } \\
\text { der mil.-techn. Akad. }\end{array}$ & 24. II. & 27.II. & - & $\begin{aligned} 1 . \\
\text { III. }\end{aligned}$ & 5 & $\begin{array}{l}\text { Der Fall wird in } \\
\text { einer späteren Arbeit } \\
\text { ausfuhrl. besprochen. }\end{array}$ \\
\hline 15 & $\begin{array}{l}\text { Alois Lustinsky, Feld- } \\
\text { webel des mil-geogr. } \\
\text { Instituts. }\end{array}$ & $\begin{array}{l}29 . \\
\text { XII. } \\
1900\end{array}$ & $\begin{array}{l}23 . \text { III. } \\
1901\end{array}$ & 12. XI. & - & 318 & $\begin{array}{l}\text { Tuberkulöse Karies } \\
\text { des Sobläfenbeins. } \\
\text { Entfernung großer } \\
\text { Teile des erkrankten } \\
\text { Knochen. Soweit ge- } \\
\text { bessert, daß er wieder } \\
\text { zu seinem Dienste. } \\
\quad \text { tauglich ist. }\end{array}$ \\
\hline
\end{tabular}


Ausräumung sämtlicherMittelohrräume im Jahre 1902.

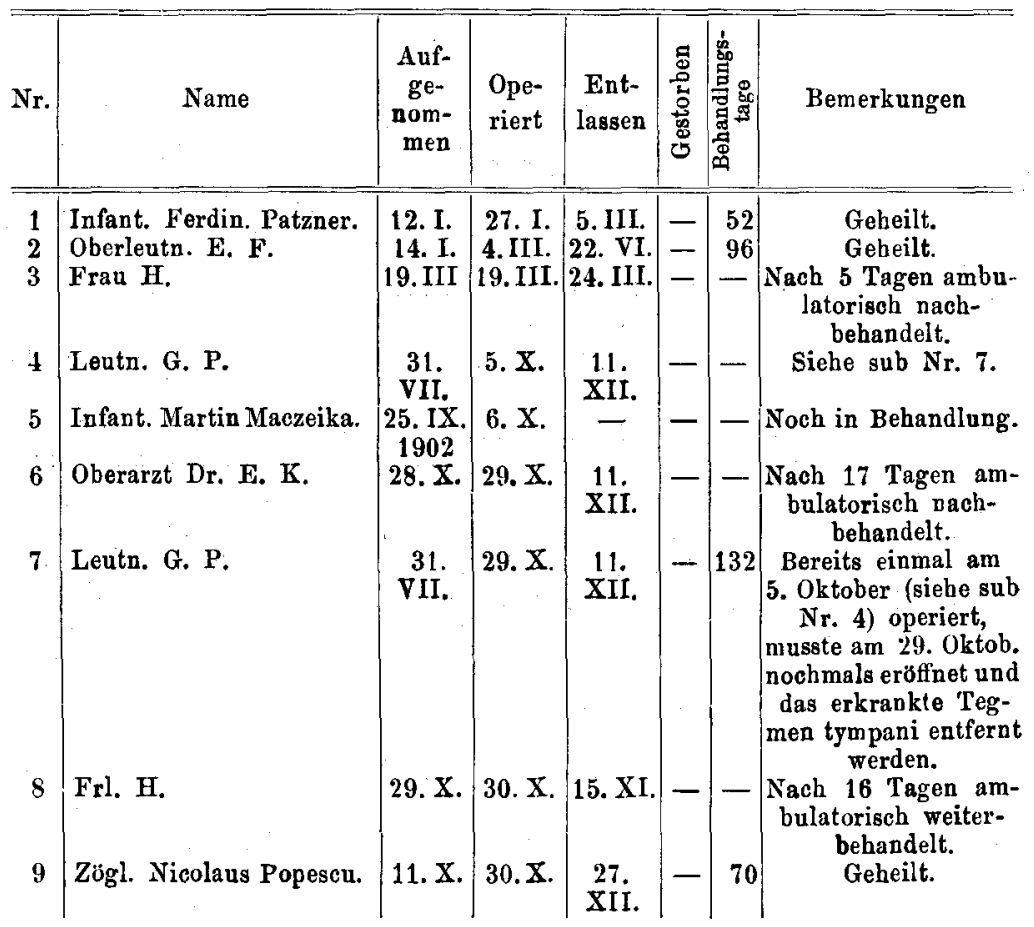

Eröffnung eines perisinuösen Abszesses.

\begin{tabular}{c|c|c}
\hline Name & Diagnose & Anmerkung \\
\hline \hline Sektionsrat N. v. W. & $\begin{array}{l}\text { Perisinuöser Abszeß an } \\
\text { der Übergangsstelle des Si-- aufenthal in in häholiche } \\
\text { nus transversus in den Sinus } \\
\text { sigmoideus. }\end{array}$ & $\begin{array}{l}\text { Nach } 5 \text { tägigem Spitals- } \\
\text { Pflege ubergeben. } \\
\text { Operiert am 21. XI. }\end{array}$
\end{tabular}

Trepanation der Schädelhöhle.

\begin{tabular}{c|c|c}
\hline \hline Name & Diagnose & Anmerkung \\
\hline \hline Infant. Ferdin. Patzner & Encephalitis diffusa, & $\begin{array}{c}\text { Aufgetreten nach Mittel- } \\
\text { ohreiterung. Die Operation } \\
\text { am 9. VI. } \\
\text { Der Mann starb am 10.VI. } \\
\text { Pathol.-anatom. Diagnose: } \\
\text { Encephalitis diffusa. } \\
\text { Der Fall wird in einer } \\
\text { säteren Arbeit besprochen. }\end{array}$ \\
&
\end{tabular}

